\title{
miR-181b-5p suppresses starvation-induced cardiomyocyte autophagy by targeting Hspa5
}

\author{
LIUHUI CHANG*, XIAOMING CHAI*, PEIMING CHEN, JIANFANG CAO, HONG XIE and JIANG ZHU
}

\begin{abstract}
Department of Anesthesiology, The Second Affiliated Hospital of Soochow University, Suzhou, Jiangsu 215004, P.R. China
\end{abstract}
Received June 28, 2018; Accepted October 16, 2018

DOI: $10.3892 / \mathrm{ijmm} .2018 .3988$

\begin{abstract}
This study aimed to investigate the role of microRNA-181b-5p (miR-181b-5p) in starvation-induced cardiomyocyte autophagy by targeting heat shock protein family A member 5 (Hspa5). For this purpose, H9c2 cardiomyocytes and neonatal rat ventricular myocytes (NRVMs) were glucose-starved in Earle's Balanced Salt Solution (EBSS) for different periods of time (0, 2, 4, 6 and $8 \mathrm{~h})$. RT-qPCR analysis was performed to examine the expression of $\mathrm{miR}-181 \mathrm{~b}-5 \mathrm{p}$ in the different groups. Immunofluorescence was performed to detect the expression of LC3. In addition, the H9c2 cardiomyocytes and NRVMs were transfected with miR-181b-5p mimic, miR-181b-5p inhibitor, siHspa5 or their respective controls. An MTT assay was performed to measure cell proliferation in the different groups. Western blot analysis was performed to determine the expression of Beclin-1, Hspa5, phosphorylated phosphoinositide 3-kinase PI3K (p-PI3K), phosphorylated Akt (p-Akt), phosphorylated mammalian target of rapamycin (p-mTOR), Bcl-2, Bax and cleaved caspase-3. Flow cytometry was performed to assess cell apoptosis. A luciferase reporter assay was performed to determine whether Hspa5 is a direct target of miR-181b-5p. The results revealed that the downregulation of miR-181b-5p promoted cell autophagy in the cardiomyocytes. Moreover, miR-181b-5p negatively regulated Beclin-1 and Hspa5. Beclin-1 is a well-known autophagy- and apoptosis-related protein. In addition, cell apoptosis was attenuated by the decreased expression of miR-181b-5p in the cardiomyocytes. Bcl-2 prevented apoptosis and autophagy by binding to Bax and Bcl-2, respectively. The upregulation of miR-181b-5p inhibited autophagy and promoted apoptosis
\end{abstract}

Correspondence to: Dr Jiang Zhu or Dr Hong Xie, Department of Anesthesiology, The Second Affiliated Hospital of Soochow University, 1055 Sanxiang Road, Suzhou, Jiangsu 215004, P.R. China E-mail: xiehong_sz@hotmail.com

E-mail: zhujiang_md@outlook.com

\section{${ }^{*}$ Co-first authorship}

Keywords:microRNA,cardiomyocytes, autophagy,heatshockprotein family A member 5, phosphoinositide 3-kinase/Akt/mammalian target of rapamycin via Hspa5. miR-181b-5p inhibition promoted p-mTOR, p-Akt and p-PI3K expression via Hspa5. The results of luciferase reporter assay also confirmed that Hspa5 is a direct target of miR-181b-5p. On the whole, the findings of this study suggest that miR-181b-5p contributes to starvation-induced autophagy and apoptosis in cardiomyocytes by directly targeting Hspa5 via the PI3K/Akt/mTOR signaling pathway.

\section{Introduction}

Cardiovascular diseases, particularly coronary heart disease, remain the leading cause of mortality worldwide (1). Thus, the prevention and treatment of cardiovascular disease is of utmost importance. Autophagy was previously reported in human heart disease in the myocardial tissue of patients with dilated cardiomyopathy (2). Autophagy plays an important role in the development of a number of cardiovascular diseases, such as myocardial ischemia and reperfusion injury, myocardial infarction, cardiac hypertrophy and heart failure (3-6). Autophagy removes old or excessive cell contents from cardiomyocytes (7). Therefore, autophagy plays an important role in the survival and functions of cardiomyocytes.

Autophagy refers to the process of degrading protein macromolecules and organelles in eukaryotic cells in autophagy lysosomes, and is divided into macroautophagy, microautophagy and chaperone-mediated autophagy (CMA). $\mathrm{cmA}$ refers to the translocation of unfolded proteins into lysosomes through heat shock protein 70 (HSP70). Heat shock protein family A member 5 (Hspa5), also known as binding immunoglobulin protein $(\mathrm{BiP})$ or glucose-regulated protein 78 (GRP-78), is a member of the HSP70 family (8), and is an endoplasmic reticulum (ER) stress-associated protein which has an effect on cell protection (9). Protein synthesis in the center of ER is altered under starvation conditions so that unfolded and misfolded proteins accumulate and result in ER stress (10). This finally results in cell autophagy (11). Hspa5 is associated with autophagy and generally cardiac protection, which is expressed as an ER stress chaperone synchronously with LC3II $(12,13)$.

MicroRNAs (miRNAs or miRs) are small non-coding RNAs comprising 18-25 nucleotides and are the central regulatory factors at the post-transcriptional level in animals and plants. miRNAs bind to the 3 '-untranslated region (3'-UTR) of their target mRNAs and negatively regulate gene expression by accelerating mRNA degradation or inhibiting 
mRNA translation (14). Several miRNAs have been shown to affect autophagy and therefore control important processes that contribute to cardiovascular diseases. For example, the knockdown of miR-122 has been reported to protect $\mathrm{H} 9 \mathrm{c} 2$ cardiomyocytes from hypoxia-induced apoptosis and to promote autophagy (15). It has also been shown that miR-365 has the ability to accelerate cardiac hypertrophy by inhibiting autophagy via the modulation of Skp2 expression (5). miR-181b-5p may also suppress proliferation, migration and invasion and promote apoptosis in astrocytoma (16). miR-181b-5p expression has also been shown to be associated with asthma (17) and schizophrenia (18). However, the role of miR-181b-5p in cardiomyocyte autophagy remains unknown.

In the present study, the role of miR-181b-5p in cardiomyocyte autophagy was investigated. Hspa5 was predicted to be a direct target of miR-181b-5p by bioinformatics analysis. The present study further determined that the downregulation of miR-181b-5p in starvation-induced cardiomyocyte autophagy may be due to the targeting of Hspa5 via the phosphoinositide 3-kinase/Akt/mammalian target of rapamycin (mTOR) signaling pathway, which plays a crucial role in cardiomyocyte protection.

\section{Materials and methods}

Cell culture and treatment. The H9c2 cell line was obtained from the Cell Line Bank of the Type Culture Collection of the Chinese Academy of Sciences (Shanghai, China) and cultured in high glucose Dulbecco's modified Eagle's medium (DMEM; Gibco/Thermo Fisher Scientific, Inc., Waltham, MA, USA) supplemented with $10 \%$ fetal bovine serum (FBS; Gibco/Thermo Fisher Scientific, Inc.), at $37^{\circ} \mathrm{C}$ in $5 \% \mathrm{CO}_{2}$.

Animal experiments. All animal protocols were approved by the Experimental Animal Committee of the Second Affiliated Hospital of Suchow University, Suchow, China. Neonatal rat ventricular myocytes (NRVMs) were isolated from 2-day-old Sprague-Dawley rats (male, $n=5$; Laboratory Animal Center of Soochow University, Suchow, China). Briefly, the hearts from 2-day-old rats were aseptically removed. Their ventricles were dissected, minced and trypsinized overnight at $4^{\circ} \mathrm{C}$. The following day, the cells were dissociated with collagenase and plated for $2 \mathrm{~h}$ at $37^{\circ} \mathrm{C}$. The non-adherent cardiomyocytes were removed and plated in 24-well plates in DMEM/F-12 medium containing $10 \% \mathrm{FBS}$ and $0.1 \mathrm{~mm}$ bromodeoxyuridine (Sigma-Aldrich/Merck KGaA, Darmstadt, Germany ). A total of $1 \times 10^{5}$ cells $/ \mathrm{cm}^{2}$ were seeded in a 24 -well plate for use in further experiments. This procedure yielded cultures with a high proportion of cardiomyocytes; microscopic observations determined that $90-95 \%$ of the cells were cardiomyocytes, as assessed by the microscopic observation of cell beating. To mimic starvation, cardiomyocytes were incubated in Earle's Balanced Salt Solution (EBSS; Gibco/Thermo Fisher Scientific, Inc.) for different periods of time (0, 2, 4, 6 and $8 \mathrm{~h})$.

Cell transfection. The cells were plated into 6-well plates $\left(1 \times 10^{5}\right.$ per well) and incubated at $37^{\circ} \mathrm{C}$ for $24 \mathrm{~h}$. The cells were either left untransfected or transiently transfected with a miR-181b-5p mimic, miR-Con, miR-181b-5p inhibitor, inhibitor-Con, siHspa5 or Con-siRNA (all from Guangzhou RiboBio Co. Ltd., Guangzhou, China) using Lipofectamine 2000 (Invitrogen/Thermo Fisher Scientific, Inc.) according to the manufacturer's instructions. Once the transfection was successful, the following experiments began.

Reverse transcription-quantitative PCR (RT-qPCR). The cells were plated in 6 -well plates $\left(1 \times 10^{5}\right.$ per well). After transfecting the cells for $48 \mathrm{~h}$, total RNA was extracted from the cells using TRIzol reagent (Invitrogen/Thermo Fisher Scientific, Inc.). The concentration and purity of the RNA was measured using a NanoDrop One Microvolume UV-Vis Spectrophotometer (Thermo Fisher Scientific, Inc.) according to the manufacturer's instructions. Complementary DNA (cDNA) was synthesized using RevertAid First Strand cDNA (Fermentas, Waltham, MA, USA) or the TaqMan MicroRNA Reverse Transcription kit (Applied Biosystems, Foster City, CA, USA) according to the manufacturer's instructions, then amplified using Power SYBR ${ }^{\circledR}$-Green PCR Master Mix or TaqMan MicroRNA Assay (Applied Biosystems). The primers used were as follows: miR-181b-5p forward, 5'-ACACTCCAG CTGGGACTTGGGCACTGAAACA-3' and reverse, 5'-TGG TGTCGTGGAGTCG-3'; and U6 forward, 5'-CTCGCTTCG GCAGCACA-3' and reverse, 5'-AACGCTTCACGAATTTGC GT-3'. U6 was used for normalization.

Western blot analysis. The H9c2 cardiomyocytes and NRVMs were plated in 6 -well plates $\left(1 \times 10^{5}\right.$ per well). After transfecting the cells for $48 \mathrm{~h}$, total protein was extracted using the RIPA lysis buffer (Beyotime Institute of Biotechnology, Shanghai, China). The concentration and purity of the protein were measured using a NanoDrop One Microvolume UV-Vis Spectrophotometer(Thermo FisherScientific,Inc.) according to the manufacturer's instructions. Protein samples were separated on SDS-PAGE gels (8\%, 100-300 kDa; 10\%, 30-100 kDa; $12 \%, 10-50 \mathrm{kDa}$ ) and transferred onto polyvinylidene difluoride membranes (Millipore, Bedford, MA, USA). The protein loaded was $35 \mu \mathrm{g}$ per lane. The membranes were blocked for $2 \mathrm{~h}$ in 5\% skimmed milk at room temperature and then incubated with primary antibodies (Beclin-1, sc-48341, 1:1,000; Hspa5, sc-376768, 1:1,000; mTOR, sc-8319, 1:1,000; p-mTOR, sc-293132, 1:1,000; Akt, sc-135829, 1:1,000; p-Akt, sc-271964, 1:1,000; PI3K, sc-293172, 1:1,000; all from Santa Cruz Biotechnology; and p-PI3K,\#4228, 1:1,000; cleaved caspase-3, \#9661, 1:1,000; Cell Signaling Technology, Danvers, MA, USA; Bcl-2, sc-509, 1:1,000; Bax, sc-20067, 1:1,000; GAPDH, sc-47724, 1:1,000; Santa Cruz Biotechnology) overnight at $4^{\circ} \mathrm{C}$. The membranes were washed with TBST 3 times, and incubated with horseradish peroxidase-labeled secondary antibodies (7076; Cell Signaling Technology) for $2 \mathrm{~h}$ at room temperature, and washed with TBST 3 times. Subsequently, the blots were detected using an enhanced chemiluminescence kit and analyzed using ImageJ software.

Immunofluorescence. Cells on coverslips were fixed in 4\% paraformaldehyde for $20 \mathrm{~min}$ and permeabilized with $0.2 \%$ Triton X-100 in PBS for $10 \mathrm{~min}$, then blocked with PBS containing 2\% bovine serum albumin for $1 \mathrm{~h}$ at room temperature. Thereafter, the cells were incubated with primary antibodies (LC3, \#2775, 1:200, Cell Signaling Technology) 

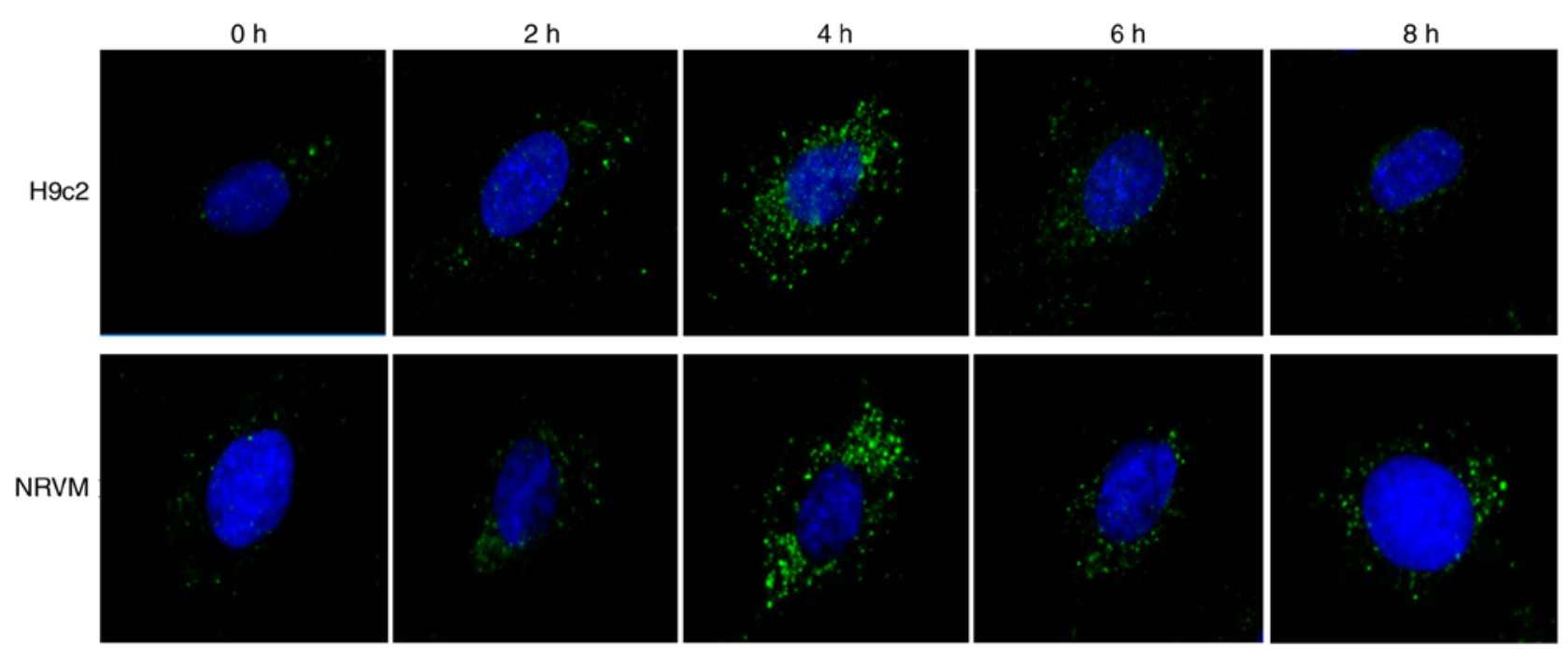

Figure 1. Immunofluorescence staining of LC3 in H9c2 cardiomyocytes and NRVMs following starvation for 0, 2, 4, 6 and 8 h. NRVMs, neonatal rat ventricular myocytes. Magnification, x200. A representative image was obtained from 3 individual experiments.
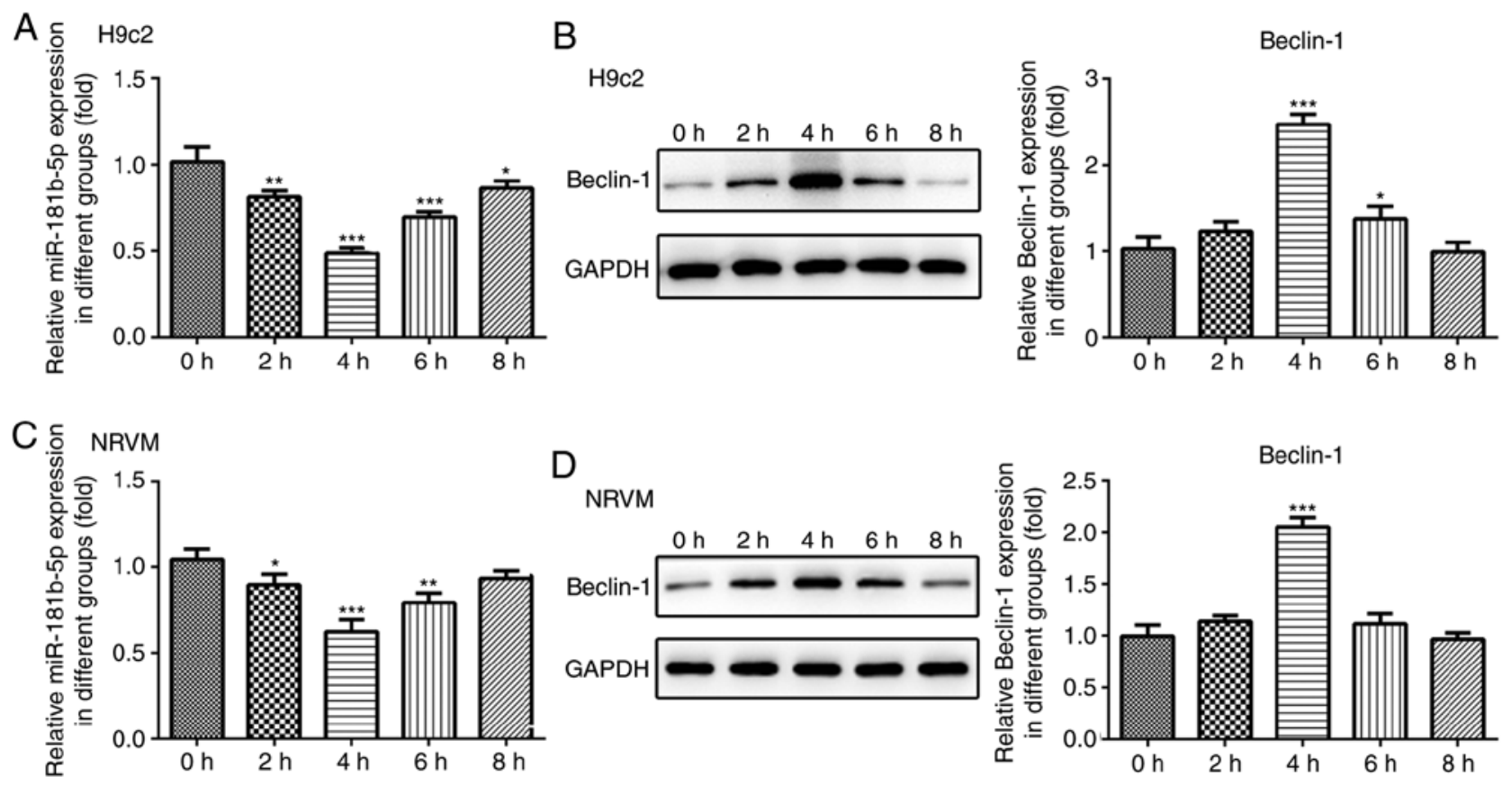

Figure 2. The expression level of miR-181b-5p and Beclin-1. (A and C) The miR-181b-5p expression level was detected by RT-qPCR in the H9c2 cardiomyocytes and NRVMs during starvation for $0,2,4,6$ and $8 \mathrm{~h}$. (B and D) The expression level of the autophagy-related protein Beclin-1 was evaluated by western blot analysis during starvation at $0,2,4,6$ and $8 \mathrm{~h} .{ }^{*} \mathrm{P}<0.05,{ }^{* * *} \mathrm{P}<0.01$ and ${ }^{* * * *} \mathrm{P}<0.001$ vs. starvation at $0 \mathrm{~h}$. NRVMs, neonatal rat ventricular myocytes.

overnight at $4^{\circ} \mathrm{C}$ and incubated with secondary antibodies (1647, 1:200, Invitrogen/Thermo Fisher Scientific, Inc.) for $2 \mathrm{~h}$ at room temperature in the dark. Finally, the cells were stained with 4',6-diamidino-2-phenylindole (DAPI; Sigma-Aldrich/ Merck KGaA) for 5 min. The coverslips were washed with PBS after each step. Images were captured using an Olympus IX50 inverted fluorescence microscope (Olympus, Tokyo, Japan).

Cell apoptosis assay. The cells were plated in 6-well plates $\left(1 \times 10^{6}\right.$ per well). After the cells were collected, they were stained using an Annexin V-PE/7-amino-actinomycin D (7-AAD) double staining kit (KeyGEN Biotech, Nanjing, China) according to the manufacturer's instructions. Cell apoptosis was quantified using FlowJo software (Tree Star, Inc., Ashland, OR, USA) on a Beckman Coulter flow cytometer (Beckman Coulter, Indianapolis, IN, USA).

Luciferase reporter assay. The potential miR-181b-5p-binding site in the 3' untranslated region (3'-UTR) of the Hspa5 gene was predicted using TargetScan (http://www.targetscan.org/ cgi-bin/targetscan/vert_71/targetscan.cgi?mirg=hsa-miR-181b-5p). $\mathrm{H} 9 \mathrm{c} 2$ cardiomyocytes were plated into 24 -well plates $\left(1 \times 10^{5} \mathrm{per}\right.$ well), and co-transfected with a pmir-GLO Dual-Luciferase miRNA Target Expression Vector (Promega Corp., Madison, WI, USA) (containing a wild-type or mutant Hspa5 3'UTR) and the miR-181b-5p mimic or miR-Con using Lipofectamine 

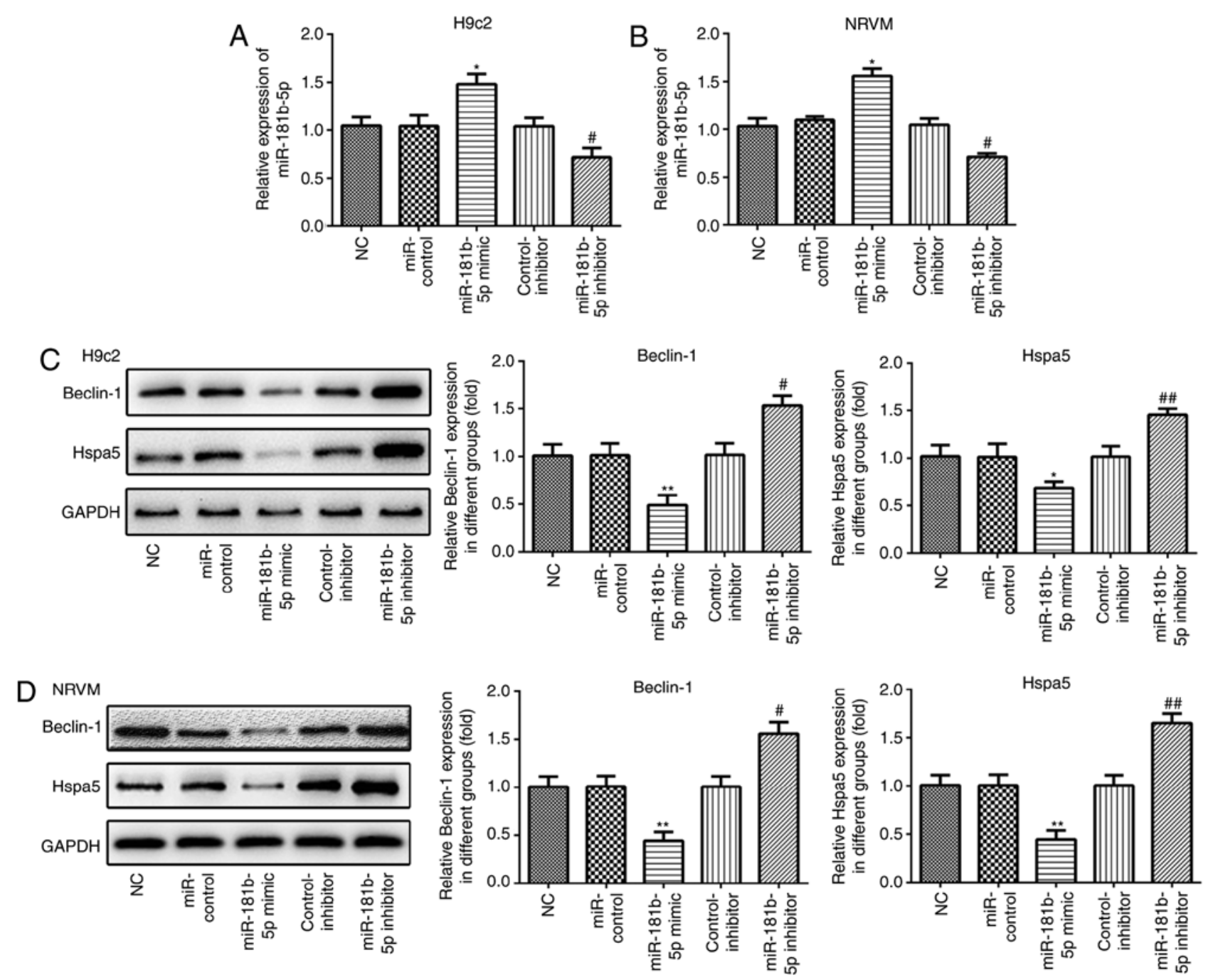

Figure 3. Beclin-1 and Hspa5 are regulated by miR-181b-5p. (A and B) H9c2 cardiomyocytes and NRVMs were transfected with miR-control, miR-181b-5p mimic, control-inhibitor and miR-181b-5p inhibitor. (C and D) The expression level of Beclin-1 and Hspa5 and its grayscale scanning analysis in H9c2 cardiomyocytes and NRVMs. NC, untransfected cells; miR-control, cells transfected with miR-181b-5p mimic control; miR-181b-5p mimic, cells overexpressing miR-181b-5p; control-inhibitor, cells transfected with miR-181b-5p inhibitor control; miR-181b-5p inhibitor, cells in which miR-181b-5p was knocked down. ${ }^{*} \mathrm{P}<0.05$ and ${ }^{* *} \mathrm{P}<0.01$ vs. NC; ${ }^{\#} \mathrm{P}<0.05$ and ${ }^{\# \#} \mathrm{P}<0.01$ vs. control-inhibitor. NRVMs, neonatal rat ventricular myocytes.

2000 (Invitrogen/Thermo Fisher Scientific, Inc.). After 48 h, the luciferase activity was measured using a Dual Luciferase Reporter Gene Assay kit (Beyotime, Shanghai, China) according to the manufacturer's instructions.

Statistical analysis. All data are represented as the means \pm standard deviation. Significant differences were determined using one-way ANOVA followed by the Tukey's Honestly Significant Difference test. Data were analyzed using SPSS version 20.0 software (IBM Corp., Armonk, NY, USA). Statistical significance is indicated by values of $\mathrm{P}<0.05$ or $\mathrm{P}<0.01$.

\section{Results}

Starvation triggers autophagy, as well as the suppression of miR-181b-5p expression in cardiomyocytes. To confirm that the autophagosomes were induced by starvation, immunofluorescence was performed in $\mathrm{H} 9 \mathrm{c} 2$ cardiomyocytes and NRVMs cultured with EBSS for different time periods of time. As shown in Fig. 1, the number of LC3-GFP-positive vesicles increased in the starved cardiomyocytes, particularly after $4 \mathrm{~h}$ of starvation. Additionally, the protein expression levels of Beclin-1 and Hspa5 were significantly upregulated in the cardiomyocytes under starvation conditions after $4 \mathrm{~h}$ of starvation (Fig. 2B and D). To assess the role of miR-181b-5p in the starved H9c2 and NRVMs cardiomyocytes, RT-qPCR analysis was performed to measure the expression of miR-181b-5p. As shown in Fig. 2A and C, the expression levels of miR-181b-5p were decreased in the starved cardiomyocytes, particularly after $4 \mathrm{~h}$ of starvation. Therefore, cell autophagy was associated with miR-181b-5p. The cardiomyocytes starved for $4 \mathrm{~h}$ were selected as the negative control (NC) for the subsequent experiments.

miR-181b-5p regulates Beclin-1 and Hspa5 expression in the cardiomyocytes under starvation conditions. RT-qPCR was performed to measure the expression of miR-181b-5p in the transfected cardiomyocytes. As shown in Fig. 3A and B, the expression of miR-181b-5p was markedly upregulated in the miR-181b-5p mimic group and downregulated in the 


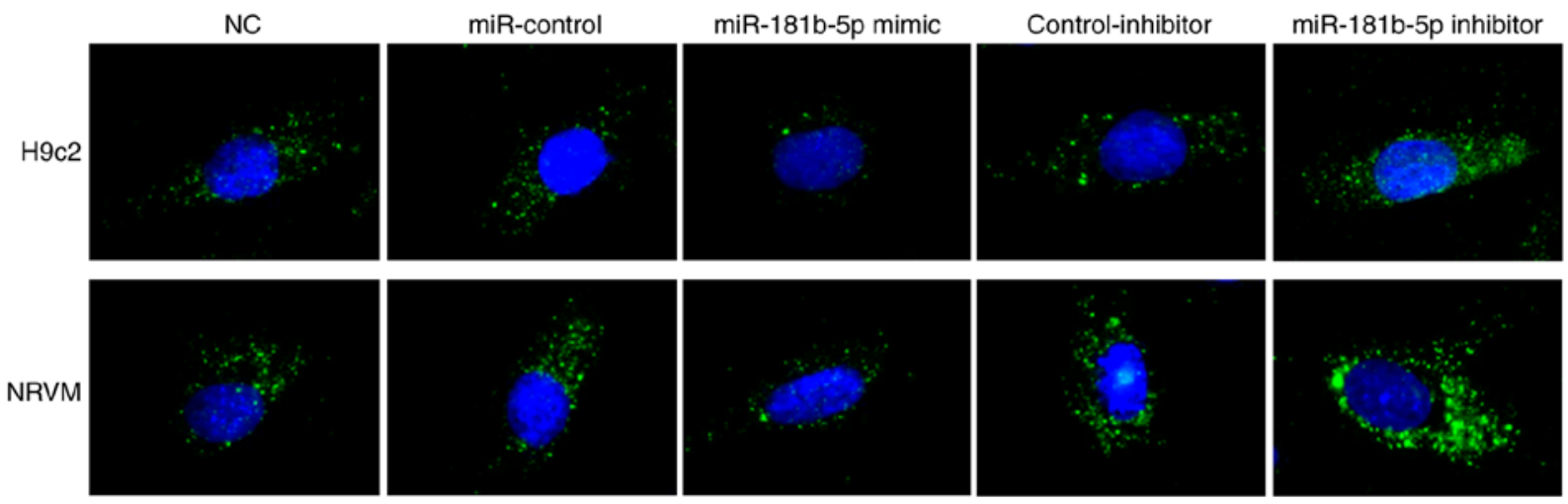

Figure 4. Immunofluorescence of LC3 in H9c2 cardiomyocytes and NRVMs following transfection with miR-control, miR-181b-5p mimic, control-inhibitor and miR-181b-5p inhibitor vs. no transfection. Magnification, x200. A representative image was obtained from 3 individual experiments. NC, untransfected cells; miR-control, cells transfected with miR-181b-5p mimic control; miR-181b-5p mimic, cells overexpressing miR-181b-5p; control-inhibitor, cells transfected with miR-181b-5p inhibitor control; miR-181b-5p inhibitor, cells in which miR-181b-5p was knocked down. NRVMs, neonatal rat ventricular myocytes.
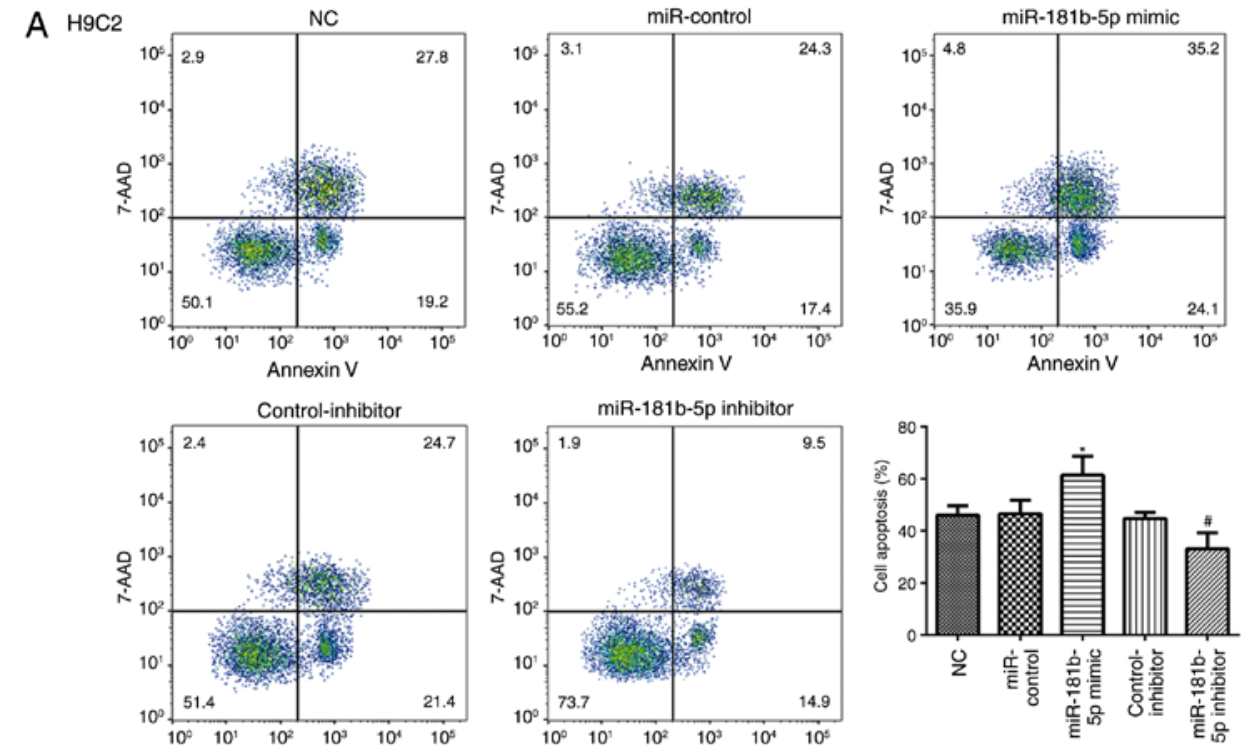

B NRVM
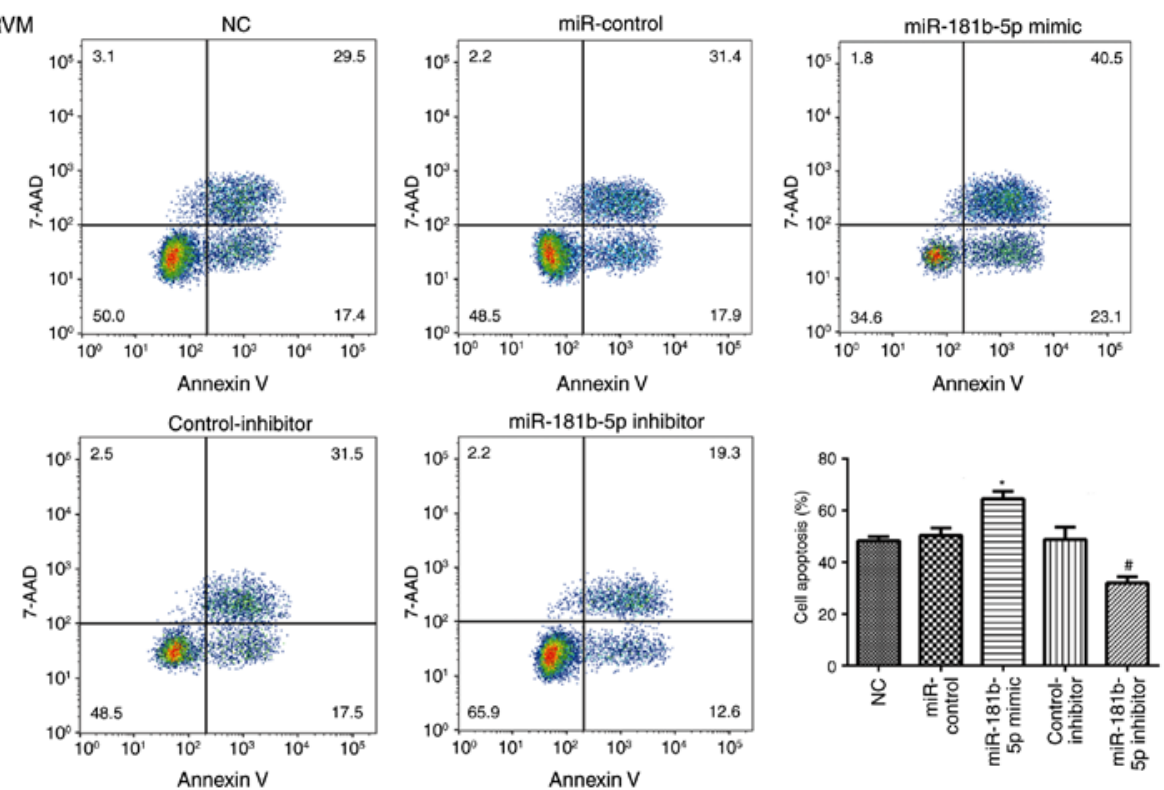

Figure 5. (A and B) miR-181b-5p regulated cell apoptosis in cardiomyocytes. Cell apoptosis was detected in H9c2 cardiomyocytes and NRVMs following transfection with miR-control, miR-181b-5p mimic, control-inhibitor and miR-181b-5p inhibitor versus no transfection. The data are shown as the means \pm SD of 3 independent experiments performed in triplicate. NC, untransfected cells; miR-control, cells transfected with miR-181b-5p mimic control; miR-181b-5p mimic, cells overexpressing miR-181b-5p; control-inhibitor, cells transfected with miR-181b-5p inhibitor control; miR-181b-5p inhibitor, cells in which miR-181b-5p was knocked down. " $\mathrm{P}<0.05$ vs. NC; ${ }^{\prime} \mathrm{P}<0.05$ vs. inhibitor control. NRVMs, neonatal rat ventricular myocytes. 

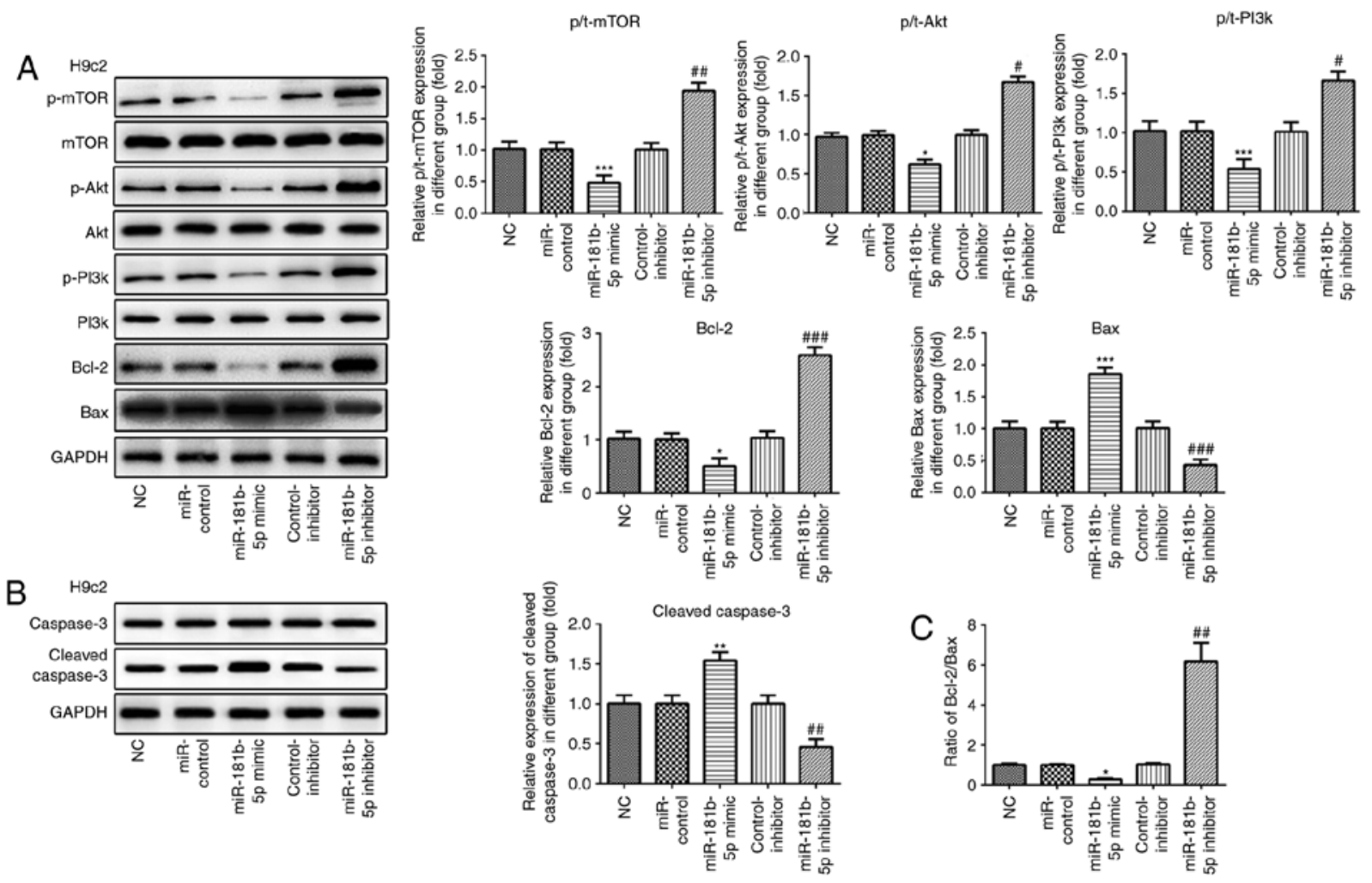

Figure 6. The PI3K/Akt/mTOR signaling pathway and apoptosis-related proteins are regulated by miR-181b-5p in H9c2 cardiomyocytes. (A and B) The protein expression of p-mTOR, mTOR, p-AKT, AKT, p-PI3K, PI3K, Bcl-2, Bax, caspase-3, cleaved caspase-3 and their grayscale scanning analysis. (C) The relative expression ratio of Bcl-2/Bax. NC, untransfected cells; miR-control, cells transfected with miR-181b-5p mimic control; miR-181b-5p mimic, cells overexpressing miR-181b-5p; control-inhibitor, cells transfected with miR-181b-5p inhibitor control; miR-181b-5p inhibitor, cells in which miR-181b-5p was knocked down. ${ }^{*} \mathrm{P}<0.05,{ }^{* *} \mathrm{P}<0.01$ and ${ }^{* * * *} \mathrm{P}<0.001$ vs. NC; ${ }^{\#} \mathrm{P}<0.05,{ }^{\# \#} \mathrm{P}<0.01$ and ${ }^{\# \# \#} \mathrm{P}<0.001$ vs. inhibitor control.

miR-181b-5p inhibitor group, compared with their respective control groups. In accordance with the results of western blot analysis, the overexpression of miR-181b-5p inhibited the expression of Beclin-1 and Hspa5, while the inhibition of miR-181b-5p promoted the expression of these proteins in the starved H9c2 cardiomyocytes (Fig. 3C) and NRVMs (Fig. 3D). These data suggest that miR-181b-5p regulates Beclin-1 and Hspa5 expression in starved $\mathrm{H} 9 \mathrm{c} 2$ cardiomyocytes and NRVMs.

miR-181b-5p regulates starvation-induced cardiomyocyte autophagy. To determine the role of miR-181b-5p in autophagy, immunofluorescence was performed to observe the formation of autophagosomes by detecting LC3B in transfected cardiomyocytes. As shown in Fig. 4, no marked differences were observed between the NC, miR-Con and inhibitor-Con groups. The miR-181b-5p mimic group exhibited markedly reduced autophagy compared with the miR-Con group. However, the inhibition of miR-181b-5p in the starved H9c2 cardiomyocytes and NRVMs markedly promoted autophagy compared with the inhibitor-Con group. These data indicated that the downregulation of miR-181b-5p promoted autophagy in the starved H9c2 cardiomyocytes and NRVMs.

miR-181b-5p regulated cell apoptosis in starved cardiomyocytes. To explore the role of miR-181b-5p in cell apoptosis, flow cytometry and western blot analysis were performed to measure cell apoptosis in the transfected cardiomyocytes. As shown in Fig. 5, no significant differences were observed in the apoptosis of the cells in the NC, miR-control and control-inhibitor. However, the overexpression of miR-181b-5p in the starved H9c2 cardiomyocytes and NRVMs significantly promoted cell apoptosis compared with the miR-Con group. However, the inhibition of miR-181b-5p in the starved H9c2 cardiomyocytes and NRVMs significantly inhibited cell apoptosis compared with the inhibitor-Con group. The results of western blot analysis also revealed that the overexpression of miR-181b-5p inhibited the protein expression levels of $\mathrm{Bcl}-2$, while it increased the protein expression levels of Bax and cleaved caspase-3. Additionally, the inhibition of miR-181b-5p enhanced the protein expression levels of Bcl-2, while it decreased the protein expression levels of Bax and cleaved caspase-3 (Figs. 6A and B, and 7A and B). The $\mathrm{Bcl}-2 / \mathrm{Bax}$ ratio was similar to the trend observed with the expression of Bcl-2 (Figs. 6C and 7C). These data indicated that miR-181b-5p regulated cell apoptosis in the starved $\mathrm{H} 9 \mathrm{c} 2$ cardiomyocytes and NRVMs.

miR-181b-5p regulates the PI3K/Akt/mTOR signaling pathway in the starved cardiomyocytes. Western blot analysis was carried out to examine the effects of miR-181b-5p on the PI3K/Akt/mTOR signaling pathway. As shown in Figs. 6 and 7, the overexpression of miR-181b-5p in the starved H9c2 cardiomyocytes and NRVMs significantly decreased the protein expression levels of p-mTOR, p-Akt and p-PI3K, compared with the miR-Con group. However, the inhibition of 
A
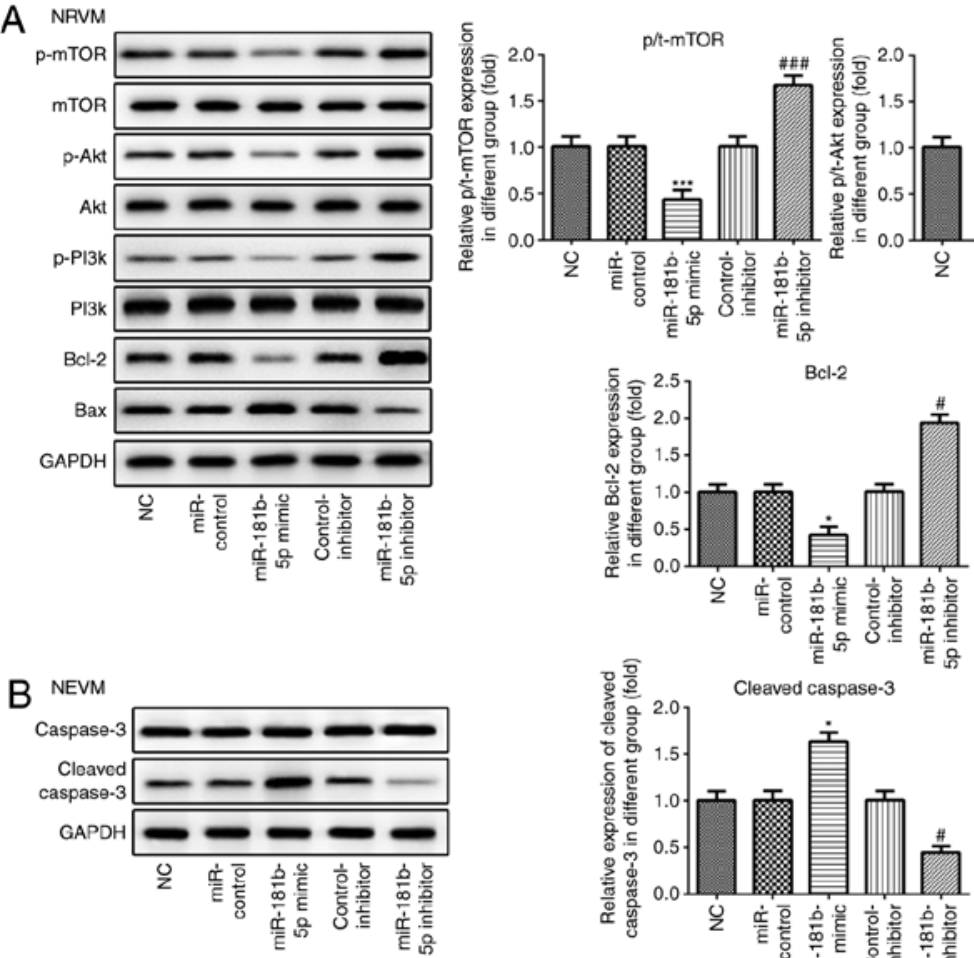

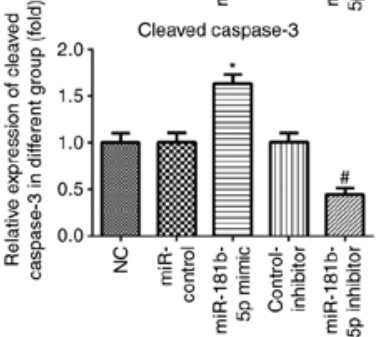

$p / t-P 13 k$

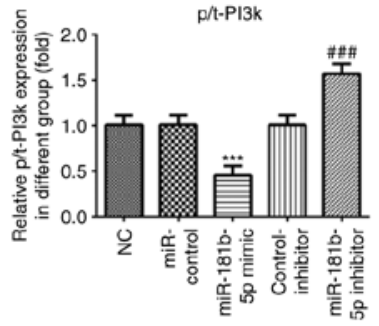

Bax
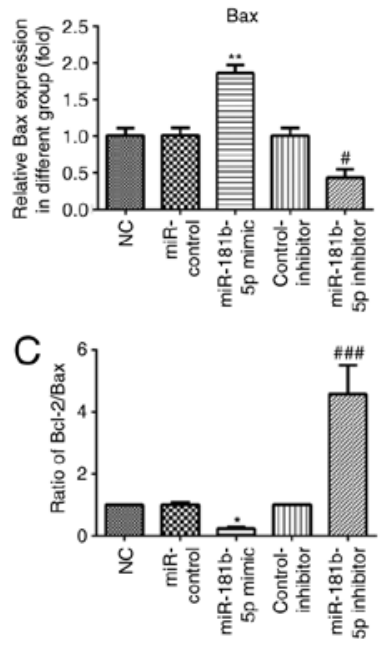

Figure 7. The PI3K/Akt/mTOR signaling pathway and apoptosis-related proteins are regulated by miR-181b-5p in NRVMs. (A and B) The protein expression of p-mTOR, mTOR, p-AKT, AKT, p-PI3K, PI3K, Bcl-2, Bax, caspase-3, cleaved caspase-3 and their grayscale scanning analysis. (C) The relative expression ratio of Bcl-2/Bax. NC, untransfected cells; miR-control, cells transfected with miR-181b-5p mimic control; miR-181b-5p mimic, cells overexpressing miR-181b-5p; control-inhibitor, cells transfected with miR-181b-5p inhibitor control; miR-181b-5p inhibitor, cells in which miR-181b-5p was knocked down. ${ }^{*} \mathrm{P}<0.05,{ }^{* *} \mathrm{P}<0.01$ and ${ }^{* * * *} \mathrm{P}<0.001$ vs. NC; ${ }^{*} \mathrm{P}<0.05$ and ${ }^{\# \# \#} \mathrm{P}<0.001$ vs. inhibitor control. NRVMs, neonatal rat ventricular myocytes.

miR-181b-5p in the starved H9c2 cardiomyocytes and NRVMs significantly increased the protein expression levels of p-mTOR, p-Akt and p-PI3K, compared with the inhibitor-Con group. These results indicated that miR-181b-5p regulated the PI3K/Akt/mTOR signaling pathway in the starved H9c2 cardiomyocytes and NRVMs.

$H s p a 5$ is a direct target of $m i R-181 b-5 p$. To elucidate the mechanisms through which miR-181b-5p regulates autophagy and apoptosis in starved H9c2 cardiomyocytes and NRVMs, the potential targets of miR-181b-5p were investigated using TargetScan, which identified the 3'-UTR region of Hspa5 mRNA as a match to miR-181b-5p. According to a previous study, Hspa5 participates in cardiac protection via autophagy (19). Thus, in this study, a dual-luciferase reporter assay was performed to examine whether Hspa5 is a direct target of miR-181b-5p. As shown in Fig. 8, the miR-181b-5p mimic significantly inhibited the dual-luciferase activity of the 3'UTR-WT of Hspa5 compared with the NC group. These data suggest that Hspa5 is a direct target of miR-181b-5p and that miR-181b-5p negatively regulates Hspa5.

siHspa5 inhibits autophagy in starved cardiomyocytes. RT-qPCR and western blot analysis were performed to measure the expression levels of Hspa5 in the transfected cardiomyocytes. As shown in Fig. 9A and B, no significant difference was observed in the expression of $\mathrm{Hspa} 5$ between the NC and siRNA-Con group; however, the mRNA and protein expression levels of Hspa5 were downregulated in

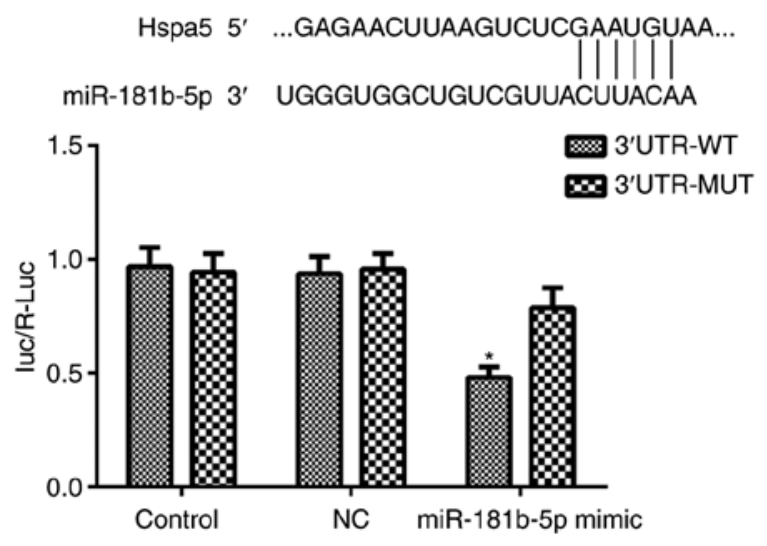

Figure 8. Association between miR-181b-5p and Hspa5. miR-181b-5p directly targeted $\mathrm{Hspa} 5$ and this was detected by luciferase reporter assay. Control, untransfected cells; NC, H9c2 co-transfected with miR-181b-5p mimic control and 3'UTR-WT or 3'UTR-MUT; H9c2 co-transfected with miR-181b-5p mimic and 3'UTR-WT or 3'UTR-MUT. "P<0.05 vs. 3'UTR WT.

the siHspa5 group, which indicated the successful transfection of siHspa5. Western blot analysis was then performed to measure Beclin-1 expression. siHspa5 inhibited the protein expression of Beclin-1 in the H9c2 cardiomyocytes (Fig. 9C) and NRVMs (Fig. 9D). These data suggest that siHspa5 regulates Beclin-1 expression in starved H9c2 cardiomyocytes and NRVMs.

To determine the role of Hspa5 in autophagy, immunofluorescence was performed to observe the autophagosomes 
A

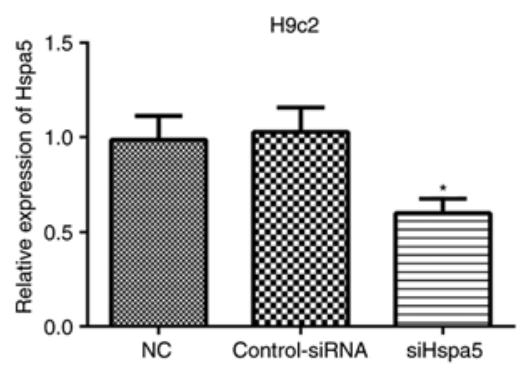

$\mathrm{B}$

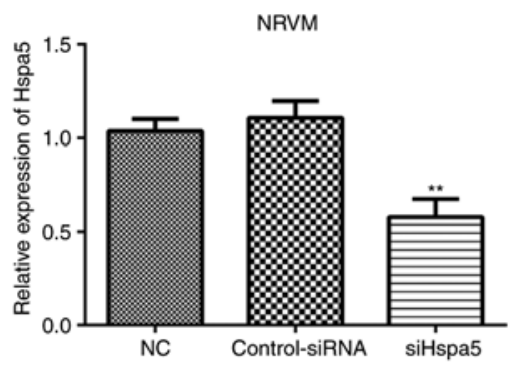

C

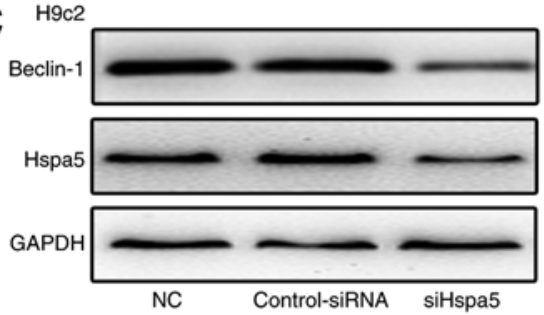

D NRVM

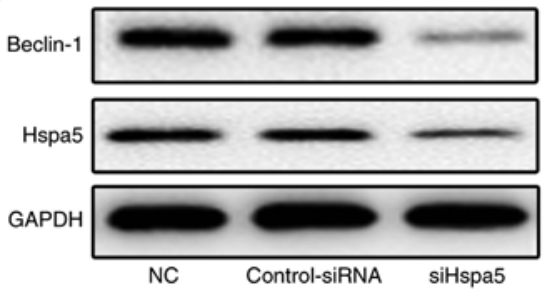

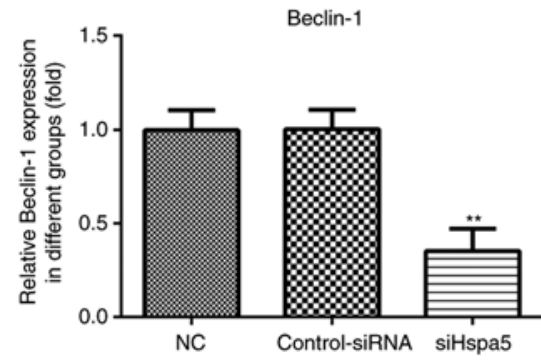
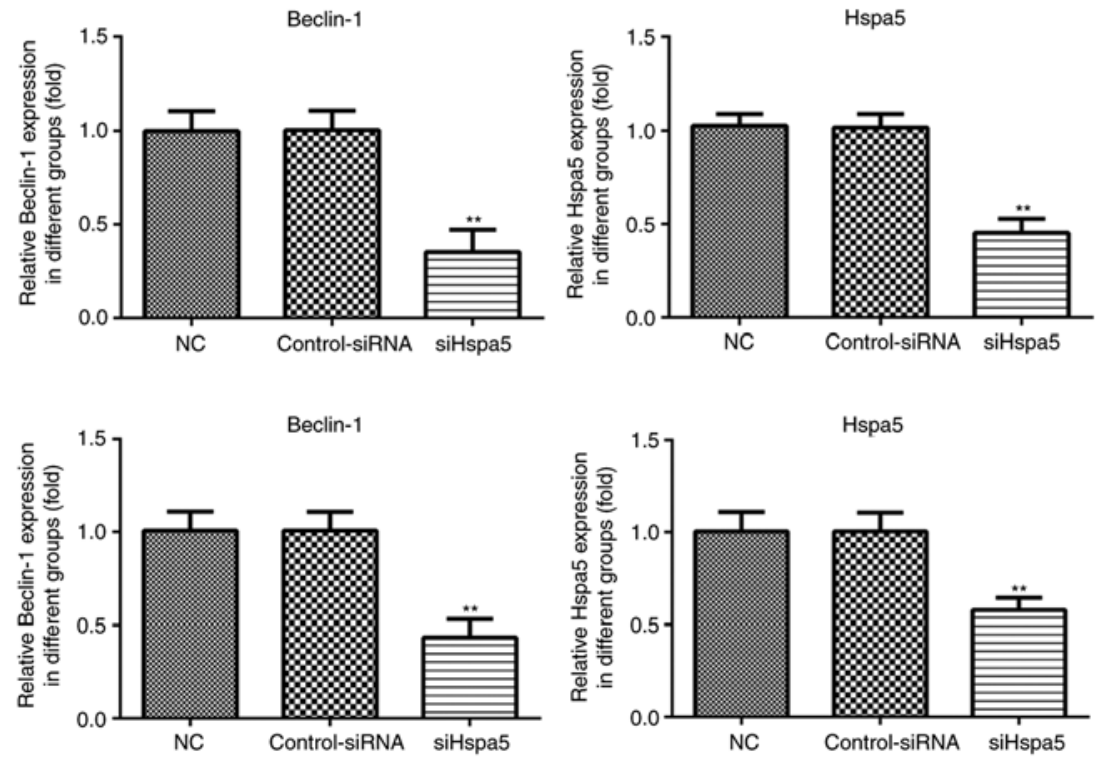

Figure 9. Beclin-1 and Hspa5 expression level are affected by Hspa5. (A and B) RT-qPCR was used to determine the transfection efficacy in H9c2 cardiomyocytes and NRVMs. (C and D) Beclin-1 and Hspa5 expression levels were detected by western blot analysis and analyzed by grayscale scanning in H9c2 cardiomyocytes and NRVMs ( $\mathrm{n}=3$ cardiomyocytes). ${ }^{*} \mathrm{P}<0.05$ and ${ }^{* *} \mathrm{P}<0.01$ vs. NC. NRVMs, neonatal rat ventricular myocytes.

in the transfected cardiomyocytes. As shown in Fig. 10, siHspa5 markedly reduced autophagy in the H9c2 and NRVMs compared with the Con-siRNA group. These data indicated that Hspa5 regulated autophagy in the starved H9c2 cardiomyocytes and NRVMs.

siHspa5 promotes the apoptosis of starved cardiomyocytes. To explore the role of Hspa5 in cell apoptosis, flow cytometry and western blot analysis were performed to measure the apoptosis of the transfected cardiomyocytes. As shown in Fig. 11, transfection with siHspa5 significantly promoted the apoptosis of H9c2 and NRVMs compared with the Con-siRNA group. Western blot analysis also revealed that siHspa5 inhibited the protein expression levels of Bcl-2, while it increased the protein expression levels of Bax and cleaved caspase-3 (Figs. $12 \mathrm{~A}$ and $\mathrm{B}$, and $13 \mathrm{~A}$ and $\mathrm{B}$ ). The Bcl-2/Bax ratio also exhibited a similar to Bcl-2 expression (Figs. 12C and 13C). These data indicated that siHspa5 induced the apoptosis of starved H9c2 and NRVMs.

siHspa5 regulates the PI3K/Akt/mTOR signaling pathway in starved cardiomyocytes. Western blot analysis was carried out to investigate the effect of Hspa5 on the PI3K/Akt/mTOR signaling pathway. As shown in Figs. 12 and 13, siHspa5 significantly decreased the protein expression of p-mTOR, p-Akt and p-PI3K in the H9c2 and NRVMs, compared with the Con-siRNA group. These results indicated that Hspa5

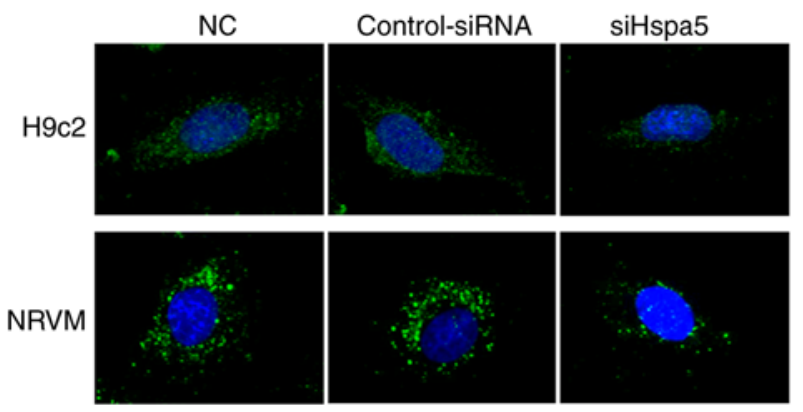

Figure 10. Immunofluorescence of LC3 in H9c2 cardiomyocytes and NRVMs following transfection with control-siRNA and si-Hspa5 both in H9c2 cells and NRVMs. The data are shown as the means \pm SD of 3 independent experiments performed in triplicate. NC, untransfected cells; control-siRNA, cells transfected with control siRNA; siHspa5, cells transfected with Hspa5 siRNA. NRVMs, neonatal rat ventricular myocytes.

regulates the PI3K/Akt/mTOR pathway in starved H9c2 cardiomyocytes and NRVMs.

\section{Discussion}

Previous studies have demonstrated that miRNAs play an essential role in cardiovascular disease. To the best of our knowledge, he current study is the first study to show the effects of miR-181b-5p on cardiomyocytes, and that the 

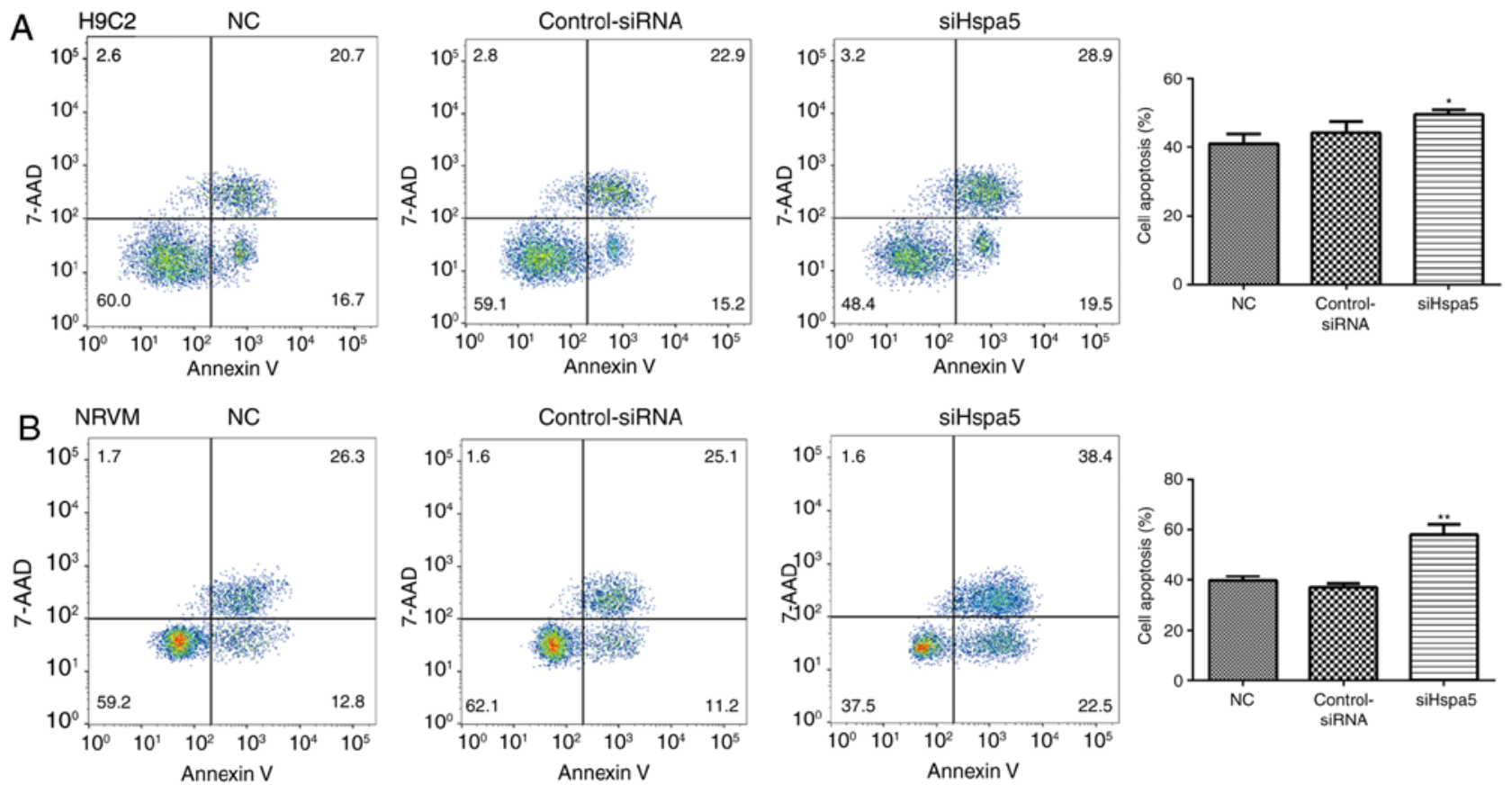

Figure 11. (A and B) Hspa5 associated with cell apoptosis in cardiomyocytes. Cell apoptosis was detected by flow cytometry in H9c2 cardiomyocytes and NRVMs following transfection with control-siRNA and si-Hspa5 both in H9c2 cells and NRVMs ( $\mathrm{n}=3$ experiments). NC, untransfected cells; control-siRNA, cells transfected with control siRNA; siHspa5, cells transfected with Hspa5 siRNA. " $\mathrm{P}<0.05$ and ${ }^{* *} \mathrm{P}<0.01$ vs. NC. NRVMs, neonatal rat ventricular myocytes.
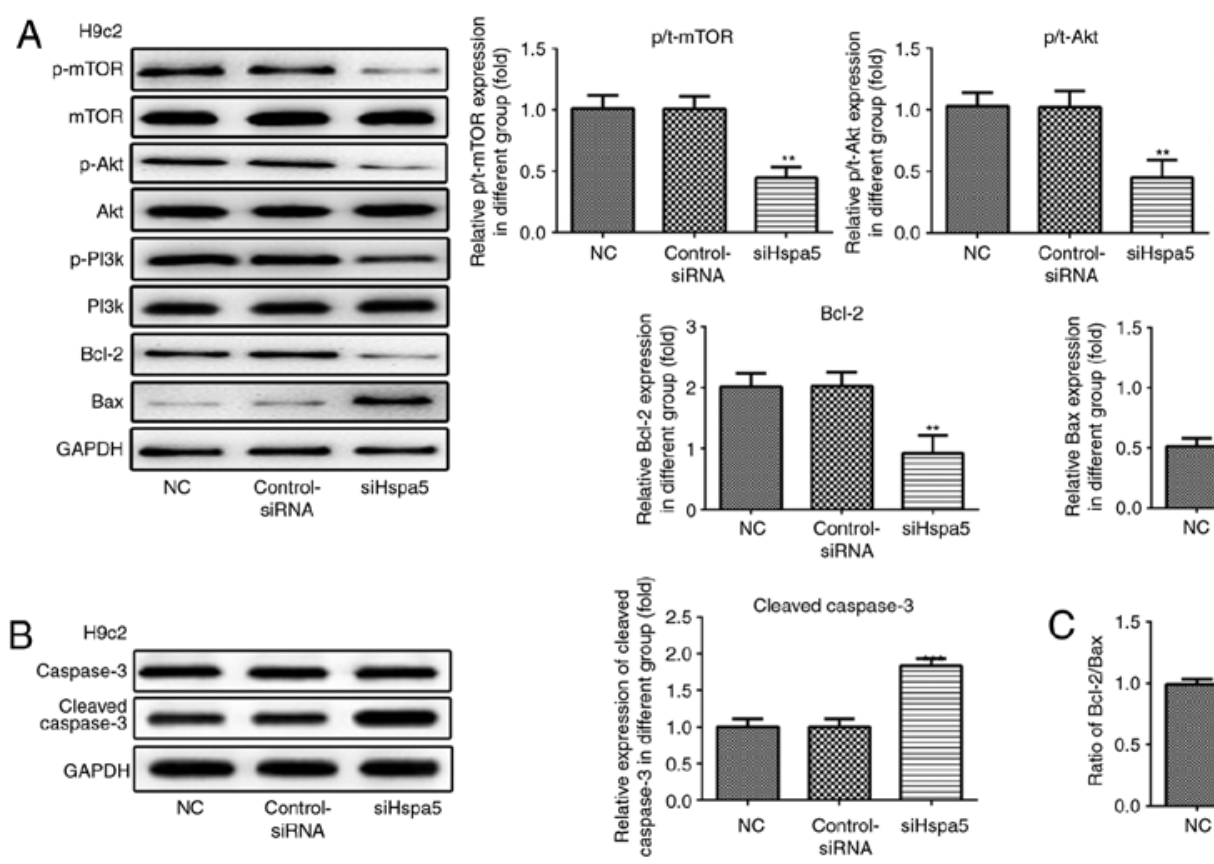

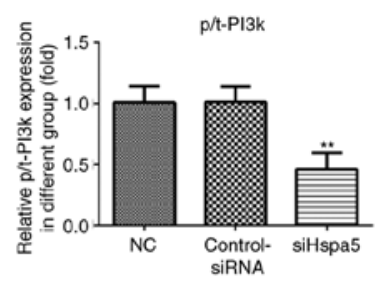

Bax
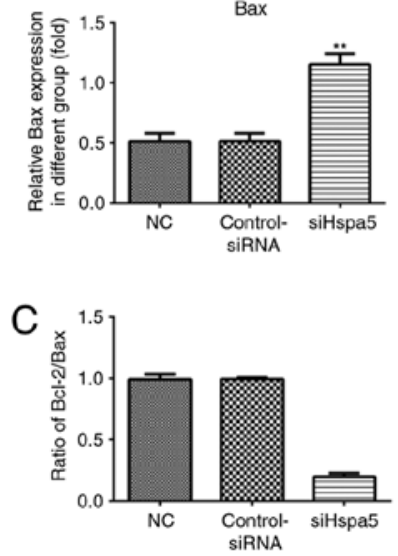

Figure 12. The PI3K/Akt/mTOR as a downstream signaling pathway of Hspa5 was inhibited and apoptosis was promoted by Hspa5 in H9c2. (A and B) Expression levels of PI3K/Akt/mTOR signaling pathway and apoptosis-related proteins, Bcl-2, Bax and caspase-3 were determined by western blot analysis and their grayscale scanning analysis ( $\mathrm{n}=3$ experiments). (C) The relative expression ratio of Bcl-2/Bax. NC, untransfected cells; control-siRNA, cells transfected with control siRNA; siHspa5, cells transfected with Hspa5 siRNA. ${ }^{* *} \mathrm{P}<0.01$ and ${ }^{* * *} \mathrm{P}<0.001$ vs. NC.

downregulation of miR-181b-5p can protect cardiomyocytes by promoting autophagy and inhibiting apoptosis. Further investigations into the underlying mechanisms indicated that autophagy and apoptosis were affected in cardiomyocytes, as miR-181b-5p directly targeted Hspa5 and that the PI3K/Akt/mTOR signaling pathway was downstream of Hspa5. Thus, the Hspa5/PI3K/Akt/mTOR signaling pathway is activated by the downregulation of $\mathrm{miR}-181 \mathrm{~b}-5 \mathrm{p}$ in starved cardiomyocytes.

Autophagy is a biological process regulated by various factors (20). Autophagy helps cells respond to various stress factors inside and outside the cell, including hunger, insulin deficiency, growth factors deficiency and endoplasmic reticulum stress (21). In this study, H9c2 cardiomyocytes 
A
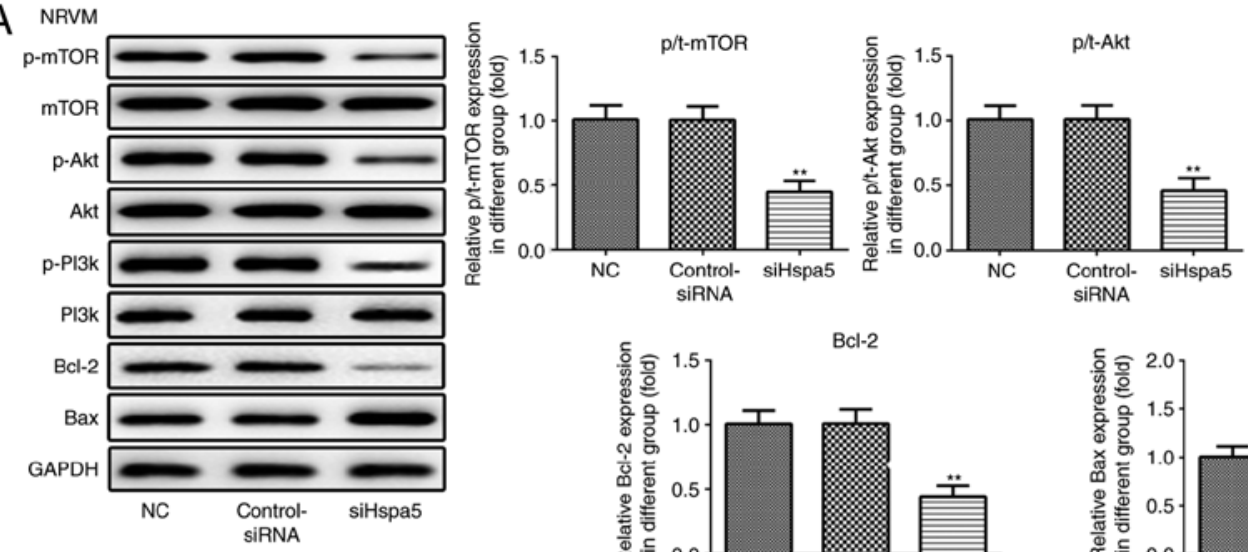

Bcl-2
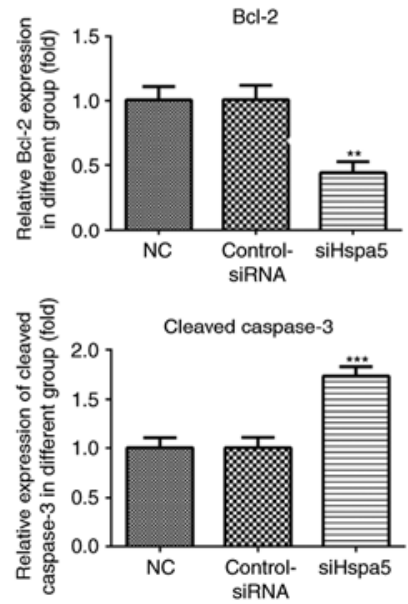

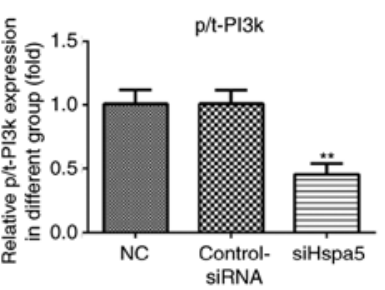

Bax
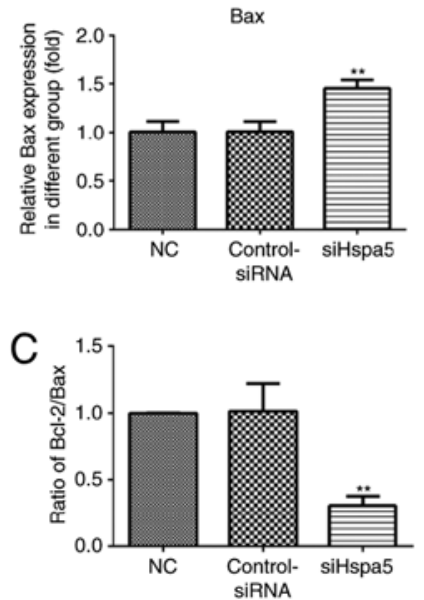

Figure 13. The PI3K/Akt/mTOR as a downstream signaling pathway of Hspa5 was inhibited and apoptosis was promoted by Hspa5 in NRVMs. (A and B) Expression levels of PI3K/Akt/mTOR signaling pathway and apoptosis-related proteins, Bcl-2, Bax and caspase-3 were determined by western blot analysis and their grayscale scanning analysis ( $n=3$ experiments). (C) The relative expression ratio of Bcl-2/Bax. NC, untransfected cells; control-siRNA, cells transfected with control siRNA; siHspa5, cells transfected with Hspa5 siRNA. ${ }^{* *} \mathrm{P}<0.01$ and ${ }^{* * * *} \mathrm{P}<0.001$ vs. NC. NRVMs, neonatal rat ventricular myocytes.

and NRVMs were cultured in EBSS to establish a starvation model. The results indicated that starvation triggered autophagosome formation, and upregulated the protein expression of Beclin-1 in H9c2 cardiomyocytes and NRVMs, particularly after $4 \mathrm{~h}$ of starvation. In addition, miR-181b-5p expression was downregulated in the starved cardiomyocytes, which revealed the possible association between miR-181b-5p and autophagy.

miR-181b-5phas been reported in several diseases, including astrocytoma, schizophrenia, pancreatic ductal adenocarcinoma and non-small lung cancer $(16,18,22,23)$. However, the effects of miR-181b-5p have not yet been evaluated to the same extent in myocardial diseases. A recent study found that miR-181b-5p expression was markedly decreased in cardiac dysfunction progression, such as diabetic cardiomyopathy (24). In addition, miR-181b-5p expression has been shown to be upregulated in heart failure resulting from cardiomyopathy (25). In this study, the effects of miR-181b-5p on cardiomyocyte autophagy and apoptosis under starvation conditions were elucidated in vitro. The results revealed that miR-181b-5p downregulation promoted cell survival by promoting autophagy and inhibiting apoptosis. Beclin-1 plays a critical role in the regulation of both autophagy and cell death (26). Hamacher-Brady et al demonstrated that Beclin-1 overexpression promoted autophagy and inhibited the apoptosis-related protein, Bax, to protect the cardiomyocytes (27). In this study, miR-181b-5p negatively regulated Beclin-1 expression and positively regulated Bax expression. It has previously been demonstrated that the increased expression levels of cleaved caspase- 3 and cleaved caspase- 9 are affected by the release of cytochrome $c$, which is related to mitochondrial apoptosis. $\mathrm{Bcl}-2$ inhibits apoptosis by binding to Bax; this binding plays a role in the formation of mitochondrial outer membrane pores so that mitochondria cannot release cytochrome $c$ (28-30). In this study, cleaved caspase- 3 and Bax were downregulated, while Bcl-2 was upregulated in cardiomyocytes transfected with miR-181b-5p inhibitors. These results indicate that the miR-181b-5p-mediated apoptosis of starved cardiomyocytes may be as a result of mitochondrial apoptosis. Thus, miR-181b-5p downregulation may promote autophagy via Beclin-1-dependent autophagy and inhibit mitochondrial apoptosis; however, further studies are required to validate this hypothesis. Cytochrome $c$ expression and mitochondrial membrane potential warrant further investigation in the future.

miRNAs act by targeting multiple genes. Hspa5, also known as GRP78, is an endoplasmic reticulum stress-related protein that plays an important role in cell protection by preventing protein aggregation (9). Hspa5 has been reported to play a role in starvation-induced cardiomyocyte autophagy (31). Since Hspa5 upregulation has been reported to be beneficial to the treatment of cardiovascular diseases, its association with miR-181b-5p was evaluated in this study. The results indicated that miR-181b-5p negatively and directly regulated Hspa5 in cardiomyocytes. Furthermore, cardiomyocytes transfected with Hspa5-siRNA exhibited a decreased autophagy and increased apoptosis. Hspa5 inhibition also downregulated the expression of Beclin-1. Based on the role of Beclin-1 in autophagy and apoptosis, it may be downstream of Hspa5, but the association between Hspa 5 and Beclin-1 requires further investigation. It may also be hypothesized that Hspa5 is a direct target of miR-181b-5p in the autophagy of starved cardiomyocytes. 
In this study, the PI3K/Akt/mTOR signaling pathway was downregulated when miR-181b-5p was overexpressed or Hspa5 was inhibited. The PI3K/Akt/mTOR signaling pathway is a recognized signaling pathway that regulates numerous biological functions in cardiomyocytes, including cell viability, apoptosis and autophagy $(32,33)$. This may be a downstream of Hspa5; however, further investigations are required.

In conclusion, in this study, the effects of miR-181b-5p and its targets on starved cardiomyocyte injury were assessed and the underlying mechanisms were revealed. miR-181b-5p mainly mediated autophagy and apoptosis. In addition, the effects of miR-181b-5p were mediated via Hspa5 and PI3K/Akt/mTOR may be the downstream signaling pathway. The PI3K/Akt/mTOR pathway signaling pathway is a well-known autophagy pathway. Animal studies of the miR-181b-5p-mediated effects may reveal the association among miR-181b-5p, autophagy and apoptosis in more detail and therefore may be performed in the future. This study demonstrates that miR-181b-5p may be a potential target in the treatment and/or prevention of cardiomyocyte injury.

\section{Acknowledgements}

Not applicable.

\section{Funding}

This study was funded by the Key Technology Application Research of Suzhou grants (SS201639 to JZ, SS201638 to JC, SS201763 to HX), the Basic Research of Medical Science of Suzhou grant (SYSD2017090 to PC) and the National Natural Science Foundation of China grant (81372024 to JZ).

\section{Availability of data and materials}

The analyzed data sets generated during the present study are available from the corresponding author on reasonable request.

\section{Authors' contributions}

LC and XC equal contributed to this article, including in the study design, data analysis and data interpretation. LC drafted the article. PC and JC collected data and analyzed the data. $\mathrm{HX}$ and $\mathrm{JZ}$ were involed in the conception and design of the study, and edited the language of the manuscript. All authors have read and approved the final manuscript.

\section{Ethics approval and consent to participate}

All animal protocols were approved by the Experimental Animal Committee of the Second Affiliated Hospital of Suchow University, Suchow, China.

\section{Patient consent for publication}

Not applicable.

\section{Competing interests}

The authors declare that they have no competing interests.

\section{References}

1. Benjamin EJ, Virani SS, Callaway CW, Chamberlain AM, Chang AR, Cheng S, Chiuve SE, Cushman M, Delling FN, Deo R, et al: Heart disease and stroke statistics-2018 update: A report from the american heart Association. Circulation 137: e67-e492, 2018.

2. Shimomura H, Terasaki F, Hayashi T, Kitaura Y, Isomura T and Suma H: Autophagic degeneration as a possible mechanism of myocardial cell death in dilated cardiomyopathy. Jpn Circ J 65: 965-968, 2001

3. Ma S, Wang Y, Chen Y and Cao F: The role of the autophagy in myocardial ischemia/reperfusion injury. Biochim Biophys Acta 1852: 271-276, 2015.

4. Wang K, Liu CY, Zhou LY, Wang JX, Wang M, Zhao B, Zhao WK, Xu SJ, Fan LH, Zhang XJ, et al: APF lncRNA regulates autophagy and myocardial infarction by targeting miR-188-3p. Nat Commun 6: 6779, 2015.

5. Wu H, Wang Y, Wang X, Li R and Yin D: MicroRNA-365 accelerates cardiac hypertrophy by inhibiting autophagy via the modulation of Skp2 expression. Biochem Biophys Res Commun 484: 304-310, 2017.

6. Takemura G, Kanamori H, Okada H, Miyazaki N, Watanabe T, Tsujimoto A, Goto K, Maruyama R, Fujiwara T and Fujiwara H: Anti-apoptosis in nonmyocytes and pro-autophagy in cardiomyocytes: Two strategies against postinfarction heart failure through regulation of cell death/degeneration. Heart Fail Rev 23: 759-772, 2018.

7. Palikaras K, Lionaki E and Tavernarakis N: Mitophagy: In sickness and in health. Mol Cell Oncol 3: e1056332, 2015.

8. Ortiz C and Cardemil L: Heat-shock responses in two leguminous plants: A comparative study. J Exp Bot 52: 1711-1719, 2001.

9. Petrovski G, Das S, Juhasz B, Kertesz A, Tosaki A and Das DK: Cardioprotection by endoplasmic reticulum stress-induced autophagy. Antioxid Redox Signal 14: 2191-2200, 2011.

10. Eizirik DL, Cardozo AK and Cnop M: The role for endoplasmic reticulum stress in diabetes mellitus. Endocr Rev 29: 42-61, 2008.

11. Yorimitsu T, Nair U, Yang Z and Klionsky DJ: Endoplasmic reticulum stress triggers autophagy. J Biol Chem 281: 30299-30304, 2006.

12. Wang L, Hong Q, Lv Y, Feng Z, Zhang X, Wu L, Cui S, Hou K, $\mathrm{Su} \mathrm{H}$, Huang $\mathrm{Z}$, et al: Autophagy can repair endoplasmic reticulum stress damage of the passive Heymann nephritis model as revealed by proteomics analysis. J Proteomics 75: 3866-3876, 2012.

13. Zhang PL, Lun M, Teng J, Huang J, Blasick TM, Yin L, Herrera GA and Cheung JY: Preinduced molecular chaperones in the endoplasmic reticulum protect cardiomyocytes from lethal injury. Ann Clin Lab Sci 34: 449-457, 2004.

14. Bartel DP: MicroRNAs: Genomics, biogenesis, mechanism, and function. Cell 116: 281-297, 2004.

15. Zhang Z, Li H, Chen S, Li Y, Cui Z and Ma J: Knockdown of MicroRNA-122 protects $\mathrm{H} 9 \mathrm{c} 2$ cardiomyocytes from hypoxia-induced apoptosis and promotes autophagy. Med Sci Monit 23: 4284-4290, 2017.

16. Zhi F, Wang Q, Deng D, Shao N, Wang R, Xue L, Wang S, Xia X and Yang Y: MiR-181b-5p downregulates NOVA1 to suppress proliferation, migration and invasion and promote apoptosis in astrocytoma. PLoS One 9: e109124, 2014

17. Huo X, Zhang K, Yi L, Mo Y, Liang Y, Zhao J, Zhang Z, Xu Y and Zhen G: Decreased epithelial and plasma miR-181b-5p expression associates with airway eosinophilic inflammation in asthma. Clin Exp Allergy 46: 1281-1290, 2016.

18. Alacam H, Akgun S, Akca H, Ozturk O, Kabukcu BB and Herken H: miR-181b-5p, miR-195-5p and miR-301a-3p are related with treatment resistance in schizophrenia. Psychiatry Res 245: 200-206, 2016.

19. Lin R, Su Z, Tan X, Su Y, Chen Y, Shu X, Liang S, Wang J and Xie S: Effect of endoplasmic reticulum stress and autophagy in the regulation of post-infarct cardiac repair. Arch Med Res, 2018.

20. Yang $Z$ and Klionsky DJ: An overview of the molecular mechanism of autophagy. Curr Top Microbiol Immunol 335: 1-32, 2009.

21. He C and Klionsky DJ: Regulation mechanisms and signaling pathways of autophagy. Annu Rev Genet 43: 67-93, 2009.

22. Tomihara H, Yamada D, Eguchi H, Iwagami Y, Noda T, Asaoka T, Wada H, Kawamoto K, Gotoh K, Takeda Y, et al: MicroRNA-181b-5p, ETS1, and the c-Met pathway exacerbate the prognosis of pancreatic ductal adenocarcinoma after radiation therapy. Cancer Sci 108: 398-407, 2017. 
23. Tian F, Shen Y, Chen Z, Li R, Lu J and Ge Q: Aberrant miR-181b-5p and miR-486-5p expression in serum and tissue of non-small cell lung cancer. Gene 591: 338-343, 2016.

24. Copier CU,Leon L, Fernandez M, Contador D and Calligaris SD Circulating miR-19b and miR-181b are potential biomarkers for diabetic cardiomyopathy. Sci Rep 7: 13514, 2017.

25. Marques FZ, Vizi D, Khammy O, Mariani JA and Kaye DM: The transcardiac gradient of cardio-microRNAs in the failing heart. Eur J Heart Fail 18: 1000-1008, 2016.

26. Zhong Y, Wang QJ, Li X, Yan Y, Backer JM, Chait BT, Heintz N and Yue Z: Distinct regulation of autophagic activity by Atg14L and Rubicon associated with Beclin 1-phosphatidylinositol-3-kin ase complex. Nat Cell Biol 11: 468-476, 2009.

27. Hamacher-Brady A, Brady NR and Gottlieb RA: Enhancing macroautophagy protects against ischemia/reperfusion injury in cardiac myocytes. J Biol Chem 281: 29776-29787, 2006.

28. Lin M, Tang S, Zhang C, Chen H, Huang W, Liu Y and Zhang J: Euphorbia factor L2 induces apoptosis in A549 cells through the mitochondrial pathway. Acta Pharm Sin B 7: 59-64, 2017.

29. Tao YW, Lin YC, She ZG, Lin MT, Chen PX and Zhang JY: Anticancer activity and mechanism investigation of beauvericin isolated from secondary metabolites of the mangrove endophytic fungi. Anticancer Agents Med Chem 15: 258-266, 2015.
30. Zhang JY, Lin MT, Tung HY, Tang SL, Yi T, Zhang YZ, Tang YN, Zhao ZZ and Chen HB: Bruceine D induces apoptosis in human chronic myeloid leukemia K562 cells via mitochondrial pathway. Am J Cancer Res 6: 819-826, 2016.

31. Chen L, Wang FY, Zeng ZY, Cui L, Shen J, Song XW, Li P, Zhao XX and Qin YW: MicroRNA-199a acts as a potential suppressor of cardiomyocyte autophagy through targeting Hspa5. Oncotarget 8: 63825-63834, 2017.

32. Hou X, Hu Z, Xu H, Xu J, Zhang S, Zhong Y, He X and Wang N: Advanced glycation endproducts trigger autophagy in cadiomyocyte via RAGE/PI3K/AKT/mTOR pathway. Cardiovasc Diabetol 13: 78, 2014.

33. Yu W, Sun H, Zha W, Cui W, Xu L, Min Q and Wu J: Apigenin attenuates adriamycin-Induced cardiomyocyte apoptosis via the PI3K/AKT/mTOR pathway. Evid Based Complement Alternat Med 2017: 2590676, 2017.

(i) (9) This work is licensed under a Creative Commons Attribution-NonCommercial-NoDerivatives 4.0 International (CC BY-NC-ND 4.0) License. 